\title{
The Health Behavior Information Needs and Preferences of Teenage and Young Adult Cancer Survivors
}

\author{
Pugh. $\mathrm{G}^{1}$; Hough.R ${ }^{2}$; Gravestock. $\mathrm{H}^{3}$ Jackson. SE ${ }^{1}$; Fisher. A $^{1}$
}

1. Health Behaviour Research Centre, University College London, 1-19 Torrington Place, WC1E 6BT

2. Department of Haematology, University College London Hospital, London, United Kingdom

3. CLIC Sargent, No 1. Farriers Yard, Assembly London, 77-85 Fulham Palace Road, London. W6 8JA

Drafted for publication in Journal of Adolescent and Young Adult Oncology

gemma.pugh.14@ucl.ac.uk

rachael.hough@uclh.nhs.uk

helen.gravestock@clicsargent.co.uk

s.e.jackson@ucl.ac.uk

abigail.fisher@ucl.ac.uk

Corresponding Author:

Gemma Pugh

Department of Epidemiology and Public Health, Health Behavior Centre

University College London

1-19 Torrington Place

WC1E 6BT

02076791736

gemma.pugh.14@ucl.ac.uk

Key words: Physical activity, diet, tobacco use, alcohol use, survivorship 


\section{Abstract}

Purpose: This study aimed to establish teenage and young adult cancer survivors (TYACS') specific interest in receiving information on physical activity, diet, smoking and alcohol consumption and their preferences regarding the delivery, format, and timing of such information.

Methods: TYACS aged 13 - 25 years were invited to complete a questionnaire assessing the advice they had received in the past and their preferences on when and how health behaviour information should be delivered.

Results: A total of 216 TYACS (mean age: 20 years; mean age at diagnosis: 16 years) completed the questionnaire. Approximately $40 \%$ of TYACS received no advice on physical activity and diet and more than half (54\%) received no advice on weight management. The majority $(>70 \%)$ reported receiving no advice on smoking or alcohol consumption. Interest in receiving lifestyle advice was high overall $(71 \%)$ but varied across behaviours, with TYACS reporting a greater level of interest in receiving advice on health protective behaviours (physical activity and diet) than health risk behaviours (smoking and alcohol consumption) ( $85 \%$ vs $\sim 15 \%$ respectively). TYACS reported seeking health behaviour information from health professionals and were most interested in information delivered online or in the form of a mobile app. Roughly equal proportions ( 18-29\%) felt health behaviour information should first be provided before, during, immediately after, and post treatment.

Conclusions: It is evident that that there is a need to develop lifestyle interventions in a range of formats available to TYACS throughout the care pathway to address the health behaviour information needs of young people with cancer. 


\section{Introduction}

Improvements in teenage and young adult (TYA) cancer care have led to a growing number of young people living with and beyond their malignant disease. ${ }^{[1-3]}$ However, it is estimated that over $90 \%$ of childhood and TYA cancer survivors (TYACS) will suffer from at least one chronic health problem within their lifetime as a result of their diagnosis and treatment. ${ }^{[4]}$ There is increasing recognition that healthy lifestyle choices such as physical activity, good dietary intake, smoking abstinence, and moderate alcohol consumption contribute to better health outcomes among young people who have been diagnosed with cancer ${ }^{[5-7]}$ However, despite the numerous benefits of healthy lifestyle behaviours, cross-sectional survey data indicate that a large proportion ( 50\%) of TYACS are inactive, ${ }^{[8,9]}$ overweight, ${ }^{[10,11]}$ regularly drink, ${ }^{[12]}$ and consume unhealthy diets low in fruit, vegetables, and key nutrients such as vitamin $D$ and calcium. ${ }^{[13-15]}$ Moreover, although the prevalence of smoking has dropped in the past decade, recent estimates indicate $22 \%$ of TYACS are current smokers. ${ }^{[16]}$ Lack of information or guidance on making healthy lifestyle may partially explain the prevalence of poor health behaviour among TYACS. In previous research TYACS have cited the absence of relevant information resources as barrier to making healthy lifestyle choices [17, 18] whilst among colorectal cancer patients ( $\mathrm{n}=15254$ ) recall of being given advice on physical activity was associated with greater odds $(\mathrm{OR}=1.90,95 \% \mathrm{Cl} 1.75-2.09) \mathrm{p}<0.001)$ of meeting the national physical activity guidelines of 30 minutes of brisk activity per day. ${ }^{[19]}$ Intervention studies conducted among TYACS have also shown promising evidence that health behaviour information, specifically risk-based information, can improve young peoples' health behaviour. ${ }^{[20]}$ In reflection of this, the provision of timely and appropriate information on health behaviour is increasingly being acknowledged as a key priority within TYA cancer care. ${ }^{[21,22]}$

Yet the health behaviour information needs of TYACS are often described as being unmet ${ }^{[9}$, ${ }^{23]}$ and in the past TYACS have explicitly described health promotion as being an 'overlooked' 
aspect of their care. ${ }^{[24]}$ One study of TYACS ( $n=74$, mean age: 22.9 years) in the United States found only $55 \%$ had received exercise information following their cancer diagnosis despite $85 \%$ indicating that they would have liked such information and support. ${ }^{[9]}$ Very little is known regarding TYACS' specific interest in, or experience of, receiving information on more sensitive health topics such as weight management, smoking abstinence, or alcohol consumption. Qualitative evidence and general reports indicate health professionals working with TYACS often face multiple barriers (e.g. time, resources, financial support, and referral options) when addressing the health behaviour needs of young people who have had cancer. $^{[25]}$ Health behaviour intervention resources, containing relevant information on physical activity, diet, smoking, and alcohol consumption have the potential to not only directly improve TYACS health behaviour, but also support health professional to deliver information and engage TYA patients in conversation on these topics.

This study aimed to establish TYACS' interest in receiving information on physical activity, diet, weight management, smoking and alcohol consumption and identify their preferences regarding the format, delivery, and timing of such information. Such data, gathered from a patient-centred perspective, is central to the development of health behaviour change resources for young people with cancer. ${ }^{[26]}$

\section{Methods}

\section{Participants \& Recruitment}

TYACS were recruited through University College Hospital, London (UCLH) or project partners CLIC Sargent (a UK cancer charity for children and young people) between May 2015 and April 2016. A Health and Lifestyle Questionnaire was advertised in patient waiting areas at University College Hospital, London (UCLH) and in the online forums and participation networks of CLIC Sargent. In alignment with the National Cancer Institute (NCI) definition [24] of cancer survivor, any young person between the age of 13 and 25 years who had been 
diagnosed with cancer at any point in their lifetime was eligible to complete the questionnaire; this included those aged 13-25 years who had been diagnosed with cancer during their childhood (aged 0-12 years). The inclusion criteria were deliberately kept broad in order to capture a wide cross-section of TYACS' views on health behaviour advice, rather than limiting to specific age or disease groups. TYACS were eligible to complete the survey regardless of their treatment status. TYACS who identified themselves as eligible had the option to complete the questionnaire either in electronic format online or in paper format. By completing and returning the survey participants were made aware they were consenting to their anonymous data being used for analysis. Ethical approval for this study was provided by University College London Research Ethics Committee reference: 6206/001 and London Hampstead Research Ethics Committee reference: 15/LO/0764.

\section{Measures}

Questions contained within the TYACS Health and Lifestyle Questionnaire were adapted from a large scale survey of adult aged cancer survivors. ${ }^{[25]}$

Demographic and health status: Participants were asked to report their age, sex, highest level of educational attainment, marital status, current living arrangement, ethnicity, type of cancer diagnosed with, date of diagnosis, current treatment status, and any health problems (other than cancer) they suffer from.

Health behaviour information and advice received: Participants were asked to report if, since being diagnosed with cancer, they had ever received information or advice on five health behaviours (physical activity, diet, weight management, smoking and alcohol consumption) from a health professional involved with their cancer treatment. Those who indicated that they had received health behaviour advice were asked to provide specific details of the advice they were given and who had provided the advice in an open response format. Participants were also asked to report whether they thought the amount of health behaviour advice they had received was too little, too much or about right. 
Health behaviour information and advice preferences: Participants were asked to report their level of interest in receiving information and advice on all health behaviour topics covered within this study. Participants were also asked to indicate whether they would have taken up the offer of health behaviour advice at the end of their initial treatment and whether they would take up the offer now. TYACS were also asked to report when they thought the best time to offer lifestyle information to young people with cancer would be and their level of interest in various advice formats. Participants were also asked to indicate the source(s) which they typically consult for health behaviour information.

\section{Statistical Analysis}

Littles Missing Completely at Random test was performed to evaluate the patterns of missing data [26]. Descriptive statistics to were used to (a) characterize participants and examine the cancer history and health status of the sample; (b) determine the proportion of TYA cancer survivors who reported receiving health behaviour advice and their views on the amount of advice they were given; and (c) gauge TYACS' interest in lifestyle information and preferences regarding information delivery. Logistic regression models were used to explore associations between participant characteristics (age, sex, age at diagnosis, treatment status, and number of health problems) and both past experience of receiving advice (received vs not received) and interest in advice (interested vs not interested) on each behaviour. Chi-Square analyses were performed to examine potential differences in TYACS preferences regarding the timing of information delivery. Open-response answers were coded line-by-line and analysed according to Elo and Kynas' (2008) process of content analysis. ${ }^{[27]}$ In total, 441 individual open response comments were analysed. Cohens Kappa was run to determine the inter-rater reliability of the content analysis. 


\section{Results}

A total of 294 TYACS started the Health and Lifestyle Questionnaire and provided at least one answer to a question contained within the survey; unfortunately due to survey drop out only 216 TYACS provided data on key variables required for this study. Within this study any residual missing data points were deemed to be missing completely at random (MCAR) as there were no systematic differences between missing and observed values $\left(X^{2}=0.00, d f=0\right.$, $\mathrm{p}>0.05$ ) indicating no key demographic differences between completers and non-completers. Any residual missing data were imputed based upon simple means; a total of 40 individual data points $(0.5 \%$ of the total data set) were imputed. There was a good level of inter-rater agreement on qualitative data (Cohens Kappa >0.6; $p<0.005)$.

\section{Sample characteristics}

Participant characteristics are displayed in Table 1. Participants $(n=216)$ had an average age of 20 years (standard deviation $(S D)=2.85$; range $13-25$ years), were predominantly female $(n=130,60 \%)$, White British $(n=183,85 \%)$ and living at home with their immediate family $(n=163,75 \%)$. Most participants $(n=91,42 \%)$ reported still being in full-time education, however $23 \%$ ( $n=49$ ) of TYACS within this study reported being either unable or too ill to work.

Table 2 outlines the health and treatment characteristics of all participants. The most common cancer diagnoses were haematological malignancies (lymphoma/ leukaemia) ( $n=126,59 \%)$, bone tumours $(n=22,10 \%)$ and soft tissue sarcomas $(n=20,9 \%)$. Average age at diagnosis was 16.8 years $(S D=4.0$; range of age at diagnosis: $0-24$ years). At the time of survey completion, the majority of respondents $(n=148,69 \%)$ had finished cancer treatment, with just under half reporting that they were more than 12 months from the end of treatment $(n=92$, $44 \%)$. Two thirds $(n=141,65 \%)$ reported suffering from one or more health problems other than their original cancer diagnosis. Table 3 displays the range of health problems suffered by participants. The most common health difficulties were extreme fatigue ( $n=66,31 \%)$ and mental health problems $(n=40,19 \%)$. 


\section{Health behaviour advice received since diagnosis}

Figure 1 displays the proportion of participants who reported receiving advice on each health behaviour since their cancer diagnosis. Approximately $40 \%$ of TYACS reported receiving no advice on physical activity and diet, most $(n=117,54 \%)$ reported receiving no advice on weight, and the majority $(\geq 70 \%)$ reported receiving no advice on smoking or alcohol consumption.

Primary treating physicians (oncologists) were the main source of information for TYACS on physical activity, smoking, alcohol consumption and weight management whilst most TYACS reported receiving advice on diet from a dietician. Participants open response answers describing the advice they had received in the past are shown in Table 4. Much of the health behaviour advice participants reported receiving was general advice about maintaining a healthy lifestyle, however many participants reported receiving specific advice that related to cancer management. Specifically, participants reported being told to remain active during and after their treatment to help with recovery and to manage cancer-related fatigue. With regard to diet, advice often centred on weight management with many participants reporting that the advice they received was predominantly about calorie intake. Most TYACS reported that they were actively discouraged from smoking and that they were advised to either not drink or reduce the amount they drink.

TYACS who reported being $\geq 18$ years of age at diagnosis were more likely to report having received advice on diet $(\mathrm{OR}=1.82,95 \% \mathrm{Cl} 1.02-3.23, \mathrm{p}=0.04)$ and weight $(\mathrm{OR}=2.61,95 \% \mathrm{Cl}$ $1.435-4.754, p=0.002)$ than those aged $<18$ years at diagnosis. Unsurprisingly, TYACS $\geq 18$ years of age at the time of the study were more likely than TYACS aged $<18$ to report receiving advice on smoking $(\mathrm{OR}=0.377,95 \% \mathrm{Cl} 0.18-0.78, \mathrm{p}=0.009)$ and alcohol consumption $(\mathrm{OR}=0.402,95 \% \mathrm{Cl} 0.18-0.85, \mathrm{p}=0.018)$. There were no other significant relationships between participant characteristics and the receipt of lifestyle advice. 
The majority ( $n=170,79 \%)$ felt that the amount of information they had received in the past was 'about right'. Only $19 \%(n=42)$ felt they had not received enough information and $2 \%(n=4)$ thought they had been given too much information.

\section{Interest in health behaviour information}

Seventy-one percent of participants $(n=153)$ reported that they would take up the offer of health behaviour information, advice and support if given the opportunity. The most commonly desired topic of advice was physical activity $(n=188,87 \%)$, closely followed by weight $(n=187$, $87 \%)$, and diet $(n=180,83 \%)$ (Figure 1). Only $18 \%(n=39)$ and $11 \%(n=24)$ of TYACS surveyed reported an interest in advice on smoking and alcohol consumption, respectively.

TYACS aged >18 were significantly less likely than 13-18 year-old TYACS to report being interested in advice on diet $(\mathrm{OR}=0.41,95 \% \mathrm{Cl} 0.18-0.89, \mathrm{p}=0.024)$ and general lifestyle $(\mathrm{OR}=0.42,95 \% \mathrm{Cl} 0.18-0.96, \mathrm{p}=0.040)$, while females in comparison to males were more likely to be interested in advice on alcohol consumption $(\mathrm{OR}=1.62,95 \% \mathrm{Cl} 0.76-3.46$, $p=0.209$ ). There were no other significant relationships between age, gender, age at diagnosis, current treatment status, number of health problems and interest in advice for each health behaviour.

\section{Sources from which TYACS seek health behaviour information}

Figure 2 outlines the range of sources TYACS report consulting for information on each health behaviour topic. TYACS typically seek information on physical activity and diet from websites online, whilst health professionals were the preferred source of information on weight, smoking, alcohol consumption and general lifestyle advice. Very few TYACS $(<15 \%)$ indicated that they would seek health behaviour information from their friends, parents, telephone helplines, or YouTube. Nonetheless, a greater proportion of TYACS reported asking for advice or information from their friends rather than their parents about smoking (11\% vs. $5 \%$ ) and alcohol consumption (15\% vs $7 \%$ ). 


\section{Preferences regarding health behaviour information delivery}

The vast majority of participants reported that they would prefer health behaviour information to be available online $(n=190,88 \%)$ or in the form of app $(n=184,85 \%)$ and indicated a preference for short information leaflets over longer booklets ( $78 \%$ vs $66 \%$ respectively). Sixtyeight percent $(n=147)$ reported that they would be interested in one individual counselling session. Group counselling sessions and a telephone call from a health professional were found to be the least popular formats of advice and information delivery.

Seventy-six percent $(n=108)$ of TYACS who had finished their cancer treatment felt that if given the opportunity they probably would have taken up the offer of information and support on health behaviour at the end of their main treatment. Table 5 displays TYACS preferences on the best time to offer health behaviour information to young people with cancer. Unsurprisingly TYACS on treatment at the time of the study reported a greater interest in receiving advice during the treatment phase of the cancer continuum, particularly before treatment begins $\left(35 \%\right.$ vs $21 \%, X^{2}(5, N=210)=17.807$, exact $\left.\mathrm{p}=0.002\right)$. No other participant characteristics were found to predict timing preference. As a collective sample, most participants $(29 \%, \mathrm{n}=62)$ felt health behaviour information and advice would be best provided immediately after treatment; a similar proportion of participants felt health behaviour information would be best provided before treatment starts $(n=56,26 \%)$, during treatment $(n=39,18 \%)$, and $3-5$ months after treatment $(n=45,21 \%)$.

\section{Discussion}

Interventions which contain relevant health behaviour information have the potential to improve the physical activity, dietary, smoking and drinking behaviours of TYACS by increasing knowledge and awareness of the importance of healthy lifestyle choices; improving positive attitude and intentions towards positive health behaviour choices, and acting as a cue to positive behaviour change. However, findings from this study indicate that TYACS often do not receive health behaviour information or advice as part of routine care, particularly on 
sensitive health topics such as weight management, smoking and alcohol consumption. TYACS were predominantly interested in information on physical activity, diet and weight management and reported seeking advice from health professionals and websites. No consensus was reached as to when information or advice on health behaviour should first be provided, with fairly equal proportions of TYACS indicating that health behaviour information should be provided before treatment, during treatment and after treatment.

TYACS' high level of interest in receiving information on physical activity, diet, and weight management is encouraging and reflects findings from previous research exploring the longterm follow up preferences and psychosocial support needs of young people with cancer. ${ }^{[9}$, 16, 28] However, the finding that very few TYACS report past experience of receiving, or any interest in receiving, information on smoking and alcohol consumption is concerning given the well-established risks these behaviours have on current and future health among TYACS. [29] Previous narrative reviews exploring the risk-taking behaviours of TYACS have acknowledged that counselling TYACS on smoking and alcohol consumption is a challenge given that experimentation with such behaviour is often a standard part of youth culture. ${ }^{\left[{ }^{00}\right]}$ A qualitative study exploring the late effects information needs of adult-aged survivors of childhood and adolescent cancer identified ambivalence to receiving health risk information as a core theme. [31] Participants in the study indicated being passive towards the importance of receiving detailed information on late effects during adolescence, and were only aware of the importance of being fully informed about health risks faced by TYACS in hindsight as adults. A similar notion may apply to information on smoking and alcohol consumption; young people with cancer may be unaware and uninterested in their need for the information on these health behaviours but receiving such information may be crucial in prompting TYACS to make and sustain positive lifestyle choices. Previous intervention studies conducted among TYA-aged childhood cancer survivors have reported intensive late-effects counselling, containing explicit information on the risks of tobacco use, to be effective at improving health knowledge and lowering intentions to smoke. ${ }^{[32]}$ As such, it is clear that despite TYACS' apparent disinterest 
in information on smoking and alcohol consumption, efforts to provide TYACS with such information should be continued.

TYACS within this study most commonly reported seeking information and support on health behaviour from either health professionals or websites. This was to be expected given the close and trusting relationships TYACS often have with health professionals ${ }^{[33]}$ and the prolific use of the internet among TYAs in general. ${ }^{[34]}$ A recent systematic review of technology-based lifestyle interventions piloted among childhood and TYACS found only six interventions that were delivered via digital mediums such as websites, mobile apps, emails, text messages or games consoles, highlighting the paucity of the research within this area. ${ }^{[35]}$ These intervention studies were generally found to be effective in promoting behaviour change and were well accepted by young people with cancer. Increasingly, digital platforms of information delivery are being acknowledged as effective strategies to reach and engage young people with cancer ${ }^{[35]}$ given the wide-age range, geographical dispersion, and multiple competing demands (e.g. school, university, work) of the TYACS population. ${ }^{[20]}$

Our finding that TYACS are interested in health behaviour information throughout the cancer care pathway from diagnosis to survivorship strengthens the rationale for developing easily accessible, readily available, health behaviour information resources for young people with cancer. However, it remains to be explored whether the precise moment at which a young person with cancer would be most receptive to health behaviour information (i.e. the teachable moment) is related to current age, age at diagnosis or treatment status. We were unable to find any association between these factors and interest in health behaviour information, suggesting that the 'teachable moment' for health promotion among TYACS may be governed by intra-individual factors. Even so, there is some evidence that health behaviour interventions delivered to TYACS may be most effective in preventing the onset and development of health problems if delivered during treatment and in the early stages of survivorship care prior to the onset of treatment-related comorbidities or noted declines in 
health behaviour. ${ }^{[36]}$ Early introductions to health promotion efforts may trigger behaviour change among some TYACS and prime intentions to change behaviour among those who are not ready or unreceptive towards lifestyle change. ${ }^{[37,38]}$

This study highlights a number of key factors that should be considered when developing health promotion interventions for TYACS. It provides a useful insight into TYACS' past experience of, and overall interest in, receiving health behaviour information. This study also presents some of the first data on how and when such information should be provided to young people with cancer. However, these results should be considered in light of several limitations. Firstly, due to the nature of recruitment it is not possible to gather data on non-responders or use data submitted by TYACS who completed only small parts of the survey. Secondly, akin to the majority of existing health behaviour interventions, ${ }^{[35,39]}$ and studies investigating the psychosocial support needs of TYACS, ${ }^{[8,20]}$ the sample within this study was predominantly white and aged between 19-25 years. The possibility of response bias within the sample is high as it is likely that those who responded to the health and lifestyle survey are those who are already engaged in health promotion efforts. As such, coupled with the cross-sectional nature of the study design, our findings may not generalize to the TYACS population as a whole. Moreover, our study only investigated the information needs and preferences of TYACS with regard to physical activity, diet, smoking, and alcohol consumption. Many young people with cancer report a need for information and support on sexual health, fertility and recreational drug use. Exploring TYACS past experience and preference for the timing, delivery and format of receiving advice on these topics is a much needed direction for future research.

The results of this study indicate TYACS are highly interested in physical activity, diet and weight management advice available in digital based formats throughout the cancer pathway. It is likely that young people with cancer require additional support from health professionals on sensitive health topics such as smoking and alcohol consumption. Our findings regarding 
TYACS' preferences on health behaviour information are important in informing the development and design of health behaviour interventions strategies for young people with cancer and may overcome some of the existing barriers TYACS face to participating in structured health behaviour change programs. ${ }^{[40-42]}$

\section{Acknowledgements}

This work was supported in part by an IMPACT Award co-funded by University College London and CLIC Sargent awarded to Gemma Pugh.

\section{Author Disclosure Statement}

No competing financial interests exist. 


\section{References}

1. Barr RD, Ferrari A, Ries L, Whelan J, Bleyer W. Cancer in adolescents and young adults: A narrative review of the current status and a view of the future. JAMA Pediatrics. 2016;170(5):495-501. doi: 10.1001/jamapediatrics.2015.4689.

2. Stark D, Bowen D, Dunwoodie E, et al. Survival patterns in teenagers and young adults with cancer in the United Kingdom: Comparisons with younger and older age groups. European journal of cancer. 2015;51(17):2643-54.

3. O'Hara C, Moran A, Whelan JS, et al. Trends in survival for teenagers and young adults with cancer in the UK 1992-2006. European Journal of Cancer. 2015;51(14):2039-48. doi: http://dx.doi.org/10.1016/j.ejca.2015.06.112.

4. Hudson MM, Ness KK, Gurney JG, et al. Clinical ascertainment of health outcomes among adults treated for childhood cancer. Jama. 2013;309(22):2371-81. Epub 2013/06/13. doi: 10.1001/jama.2013.6296. PubMed PMID: 23757085; PubMed Central PMCID: PMCPmc3771083.

5. Slater ME, Steinberger J, Ross JA, et al. Physical Activity, Fitness, and Cardiometabolic Risk Factors in Adult Survivors of Childhood Cancer with a History of Hematopoietic Cell Transplantation. Biology of Blood and Marrow Transplantation. 2015;21(7):1278-83. doi: http://dx.doi.org/10.1016/j.bbmt.2015.04.007.

6. Smith WA, Li C, Nottage KA, Mulrooney DA, et al. Lifestyle and metabolic syndrome in adult survivors of childhood cancer: a report from the St. Jude Lifetime Cohort Study. Cancer. 2014;120(17):2742-50. doi: 10.1002/cncr.28670. PubMed PMID: 25070001; PubMed Central PMCID: PMC4165406.

7. Spector DJ, Noonan D, Mayer DK, et al. Are lifestyle behavioral factors associated with healthrelated quality of life in long-term survivors of non-Hodgkin lymphoma? Cancer. 2015;121(18):334351. doi: 10.1002/cncr.29490.

Belanger, L.J., et al., Physical activity and health-related quality of life in young adult cancer survivors: a Canadian provincial survey. J Cancer Surviv, 2011. 5(1): p. 44-53.

9. Murnane, A., et al., Adolescents and young adult cancer survivors: exercise habits, quality of life and physical activity preferences. Supportive Care in Cancer, 2015. 23(2): p. 501-510.

10. Garmey, E.G., et al., Longitudinal changes in obesity and body mass index among adult survivors of childhood acute lymphoblastic leukemia: a report from the Childhood Cancer Survivor Study. J Clin Oncol, 2008. 26(28): p. 4639-45.

11. Zhang, F. and S. Parsons, Obesity in Childhood Cancer Survivors: Call for Early Weight Management. Advances in Nutrition: An International Review Journal, 2015. 6(5): p. 611-619.

12. Marjerrison, S., et al., Smoking, Binge Drinking, and Drug Use Among Childhood Cancer Survivors: A Meta-Analysis. Pediatr Blood Cancer, 2016. 
13. Demark-Wahnefried, W., et al., Survivors of childhood cancer and their guardians. Cancer, 2005. 103(10): p. 2171-80.

14. Badr, H., et al., Health-related quality of life, lifestyle behaviors, and intervention preferences of survivors of childhood cancer. J Cancer Surviv, 2013. 7(4): p. 523-34.

15. Bilariki, K., et al., Low Bone Mineral Density and High Incidences of Fractures and Vitamin D Deficiency in 52 Pediatric Cancer Survivors. Hormone Research in Paediatrics, 2010. 74(5): p. 319-327.

16. Gibson, T.M., et al., Longitudinal smoking patterns in survivors of childhood cancer: An update from the Childhood Cancer Survivor Study. Cancer, 2015. 121(22): p. 4035-4043.

17. Arroyave, W.D., et al., Childhood cancer survivors' perceived barriers to improving exercise and dietary behaviors. Oncology Nursing Forum, 2008. 35(1): p. 121-130.

18. Wu, Y.P., et al., Barriers and Facilitators of Healthy Diet and Exercise Among Adolescent and Young Adult Cancer Survivors: Implications for Behavioral Interventions. J Adolesc Young Adult Oncol, 2015. 4(4): p. 184-91.

19. Fisher, A., et al., Recall of physical activity advice was associated with higher levels of physical activity in colorectal cancer patients. BMJ Open, 2015. 5(4).

20. Pugh, G., et al., Health Behavior Change Interventions for Teenage and Young Adult Cancer Survivors: A Systematic Review. J Adolesc Young Adult Oncol, 2016. 5(2): p. 91-105.

21. Daniel, C.L., et al., Needs and Lifestyle Challenges of Adolescents and Young Adults with Cancer: Summary of an Institute of Medicine and Livestrong Foundation Workshop Clin J Oncol Nurs, 2015. 19(6): p. 675-81.

22. Nass, S.J., et al., Identifying and Addressing the Needs of Adolescents and Young Adults With Cancer: Summary of an Institute of Medicine Workshop. The Oncologist, 2015. 20(2): p. 186-195.

23. Rabin, C., et al., Intervention format and delivery preferences among young adult cancer survivors. Int J Behav Med, 2013. 20(2): p. 304-10.

24. Fern, L.A., et al., The art of age-appropriate care: reflecting on a conceptual model of the cancer experience for teenagers and young adults. Cancer Nurs, 2013. 36(5): p. E27-38.

25. Berg, C., et al., Providers' Perspectives of Survivorship Care for Young Adult Survivors of Childhood Cancer. Journal of Cancer Education, 2016. 31(1): p. 31-38.

26. Craig, P., et al., Developing and evaluating complex interventions: the new medical research council guidance. BMJ, 2008. 337

27. Elo S, Kyngäs $H$. The qualitative content analysis process. Journal of Advanced Nursing. 2008;62(1):107-15. doi: 10.1111/j.1365-2648.2007.04569.x. 
28. Barakat L, Galtieri L, Szalda D, Schwartz L. Assessing the psychosocial needs and program preferences of adolescents and young adults with cancer. Supportive Care in Cancer. 2016;24(2):82332.

29. Wilson CL, Chemaitilly W, Jones KE, et al. Modifiable Factors Associated With Aging Phenotypes Among Adult Survivors of Childhood Acute Lymphoblastic Leukemia. Journal of Clinical Oncology. 2016;34(21):2509-15. doi: 10.1200/jco.2015.64.9525.

30. Morgan S, Davies S, Palmer S, Plaster M. Sex, drugs, and rock ' $n$ ' roll: caring for adolescents and young adults with cancer. Journal of clinical oncology : official journal of the American Society of Clinical Oncology. 2010;28(32):4825-30. doi: 10.1200/JCO.2009.22.5474. PubMed PMID: 20498401.

31. Lie HC, Loge JH, Fosså SD, et al. Providing information about late effects after childhood cancer: Lymphoma survivors' preferences for what, how and when. Patient Education and Counseling. 2015;98(5):604-11. doi: http://dx.doi.org/10.1016/j.pec.2015.01.016.

32. Tyc VL, Rai SN, Lensing $S$, et al. Intervention to reduce intentions to use tobacco among pediatric cancer survivors. Journal of Clinical Oncology. 2003;21(7):1366-72.

33. Gibson F, Fern L, Whelan J, et al. A scoping exercise of favourable characteristics of professionals working in teenage and young adult cancer care: 'thinking outside of the box'. European Journal of Cancer Care. 2012;21(3):330-9. doi: 10.1111/j.1365-2354.2011.01322.x.

34. Gross EF. Adolescent Internet use: What we expect, what teens report. Journal of Applied Developmental Psychology. 2004;25(6):633-49. doi: http://dx.doi.org/10.1016/j.appdev.2004.09.005.

35. Kopp LM, Gastelum Z, Guerrero $\mathrm{CH}$, et al. Lifestyle behavior interventions delivered using technology in childhood, adolescent, and young adult cancer survivors: A systematic review. Pediatric blood \& cancer. 2016. Epub 2016/07/29. doi: 10.1002/pbc.26166. PubMed PMID: 27468131.

36. Barnes M, Plaisance E, Hanks L, Casazza K. Pre-habilitation-Promoting Exercise in Adolescent and Young Adult Cancer Survivors for Improving Lifelong Health - A Narrative Review. Cancer Research Frontiers 2016 2(1):22-32.

37. Demark-Wahnefried W, Pinto BM, Gritz ER. Promoting health and physical function among cancer survivors: potential for prevention and questions that remain. Journal of clinical oncology : official journal of the American Society of Clinical Oncology. 2006;24(32):5125-31. Epub 2006/11/10. doi: 10.1200/jco.2006.06.6175. PubMed PMID: 17093274.

38. Demark-Wahnefried W, Aziz NM, Rowland JH, Pinto BM. Riding the crest of the teachable moment: promoting long-term health after the diagnosis of cancer. Journal of clinical oncology : official journal of the American Society of Clinical Oncology. 2005;23(24):5814-30. doi: 10.1200/JCO.2005.01.230. PubMed PMID: 16043830; PubMed Central PMCID: PMC1550285.

39. Pugh G, Gravestock HL, Hough RE, et al. Health Behavior Change Interventions for Teenage and Young Adult Cancer Survivors: A Systematic Review. Journal of adolescent and young adult oncology. 2016;5(2):91-105. Epub 2016/02/13. doi: 10.1089/jayao.2015.0042. PubMed PMID: 26871647. 
40. Love B, Moskowitz MC, Crook B, et al. Defining adolescent and young adult (AYA) exercise and nutrition needs: Concerns communicated in an online cancer support community. Patient Education and Counseling. 2013;92(1):130-3. doi: http://dx.doi.org/10.1016/j.pec.2013.02.011.

41. Wright M, Bryans A, Gray K, Skinner L, Verhoeve A. Physical Activity in Adolescents following Treatment for Cancer: Influencing Factors. Leukemia research and treatment. 2013;2013:592395. doi: 10.1155/2013/592395. PubMed PMID: 24163773; PubMed Central PMCID: PMC3791599.

42. Wu YP, Yi J, McClellan J, et al. Barriers and Facilitators of Healthy Diet and Exercise Among Adolescent and Young Adult Cancer Survivors: Implications for Behavioral Interventions. Journal of adolescent and young adult oncology. 2015;4(4):184-91. Epub 2015/12/24. doi: 10.1089/jayao.2015.0028. PubMed PMID: 26697268; PubMed Central PMCID: PMCPmc4684662. 